\title{
In silico Docking Analysis of Bioactive Compounds from Stoechoespermum marginatum against Colorectal Cancer
}

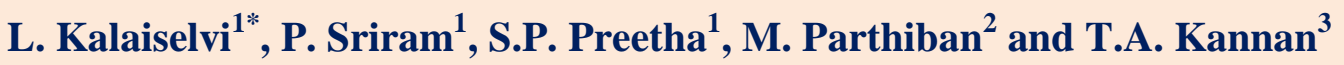 \\ ${ }^{1}$ Department of Veterinary Pharmacology and Toxicology, ${ }^{2}$ Department of Animal \\ Biotechnology, ${ }^{3}$ Department of Veterinary Anatomy, Madras Veterinary College, \\ Tamil Nadu Veterinary and Animal Sciences University, Chennai-600 007, India \\ *Corresponding author
}

\section{Keywords \\ Colorectal cancer, Stoechospermum marginatum, docking, wnt, adenomatous polyposis coli, $\beta$ - Catenin glycogen synthase kinase $3 \beta$ \\ Article Info \\ Accepted: 12 April 2019 Available Online: 10 May 2019}

\section{A B S T R A C T}

Colorectal cancer is one of the most commonly diagnosed malignancies are mainly initiated by the mutations in the wnt signalling proteins, viz., Adenomatous polyposis coli (APC), $\beta$-Catenin and glycogen synthase kinase $3 \beta$ (GSK-3 $\beta$ ). The present study focuses on molecular docking analysis of bioactive molecules isolated from Stoechospermum marginatum against wnt signalling proteins. Twelve bioactive molecules from $S$. marginatum were evaluated for their potential to interact with wnt signalling proteins. The biomolecules were screened for their in silico ADMET properties. The results revealed that compound $7(5(\mathrm{R}), 15,18(\mathrm{R} / \mathrm{S}), 19$-tetrahydroxy spata 13,16-diene) and compound 8 (19-acetoxy, 5(R), 15, 16-trihydroxy spata 13, 17-diene) had good interaction with $\beta$ catenin, APC and GSK3 $\beta$ proteins and were found to possess required ADMET criteria with good aqueous solubility, low BBB permeability, low plasma protein binding, nonhepatotoxic, non-mutagenic and lack of CYP2D6 inhibition. From the results of the study, compound 7 [5(R), 15, 18(R/S), 19-tetrahydroxy spata 13, 16-diene] and compound 8 [19acetoxy, $5(\mathrm{R}), 15$, 16-trihydroxy spata 13,17 -diene] would be a promising lead candidate for further research and development of drugs against colorectal cancer.

\section{Introduction}

Colorectal cancer (CRC) is the third most commonly diagnosed malignancy and the second leading cause of cancer-related deaths worldwide (Bray et al., 2018). The incidence of colorectal cancer continues to increase with an estimated global incidence of $10.2 \%$ in 2018 and this is expected to increase by $60 \%$ by 2030 (Arnold et al., 2016). Early stages of cancer can be readily treated by surgery whereas treatment of patient with distant metastasis and advanced stages of cancer remains challenging. Although recent advances in chemotherapy have improved management and survival of CRC patients, the side effects and development of resistance to chemotherapeutic drugs are the major limitations. The increasing incidence of CRC demands urgent need for the development of new drug molecules to overcome the low sensitivity of CRC to chemotherapeutic drugs. 
CRC is a heterogeneous disease and the development of cancer is a combined effect of both genetic alterations and environmental factors. Better understanding of molecular pathogenesis of CRC will help to develop drugs targeting specific pathways. Majority of CRC include dysregulation of wnt signalling pathway (Becer et al, 2019) and are initiated by mutations in Adenomatous polyposis coli (APC), $\beta$-Catenin and glycogen synthase kinase $3 \beta$ (GSK-3 $\beta$ ) (Blaj et al., 2017 and Naveneetha krishnan et al., 2013).

Wingless-type (Wnt) signalling is a highly conserved pathway that plays an important role in various cellular and developmental process including cellular metabolism, proliferation, differentiation, survival and apoptosis. Wnt pathway is classically divided into canonical ( $\beta$-catenin-dependent) and noncanonical ( $\beta$-catenin-independent) pathway. In canonical pathway, $\beta$-catenin acts as key modulator and wnt signalling functions by controlling the level of $\beta$-catenin in the cytoplasm. In the absence of Wnt ligands, $\beta$ catenin is degraded by a destruction complex, which contains scaffold protein Axin, APC, protein phosphatase $2 \mathrm{~A}$, GSK3 $\beta$ and casein kinase $1 \quad(\mathrm{CK} 1 \alpha)$. $\beta$-catenin is first phosphorylated by $\mathrm{CK} 1$ and GSK $3 \beta$ in the complex, which is followed by recruitment of E3 ligase $-\beta$ - TrCP for ubiquitination and proteasomal degradation. Binding of wnt ligands like Wnt3a and Wnt1 to Frizzled (FZD) receptors and low-density lipoproteinrelated protein 5/6 (LRP5/6) results in the activation of canonical pathway. Activation of receptor inhibits the activity of destruction complex either by direct interaction of Axin with LRP receptors or through recruitment of Axin binding molecule Dishevelled (Dvl). CK $1 \alpha$ and GSK3 $\beta$ in the complex phosphorylate LRP receptors which then recruit Dvl proteins to the plasma membrane where they polymerize and get activated. Activated Dvl polymers inactivate destruction complex resulting in stabilization and accumulation of $\beta$-catenin. Free cytosolic $\beta$ catenin is then translocated to the nucleus and binds with LEF (lymphoid enhancer factor) and $\mathrm{T}$ cell factor (TCF) transcription factor together with other coactivators such as cAMP-response element-binding protein (CBP) and p300 to activate the expression of Wnt target genes such as c-Myc, c-jun, cyclin $\mathrm{D}, \mathrm{PPAR} \delta$ and these genes regulates colon cell proliferation and regulation (Cheng et al., 2019; Zhan et al., 2017; Novellasdemunt et al., 2015 and Navaneethakrishnan et al., 2013). The role of Wnt signaling in colorectal carcinogenisis suggests that Wnt signaling pathway can be an effective therapeutic target for development of new drug molecules for the treatment of cancer.

Marine macroalgae, commonly known as seaweeds are rich source of bioactive compounds and produce a wide range of secondary metabolites including alkaloids, sulphated polysaccharides, flavonoids, diterpenoids, sterols (Haniya et al., 2015). The secondary metabolites produced by marine organisms are unique and structurally diverse with potentials for the development of new drug molecules. Stoechospermum marginatum (C.Agardh) Kutzung, a brown algae, is widely distributed along the coastal regions of Tamil Nadu (India) and it contains various phytochemicals such as alkaloids, glycosides, tannins, saponin, triterpenoids, flavonoids etc.

It is reported to contain antibacterial, antiproliferative, angiosuppressive, antioxidant and apoptotic activities (Anbu et al., 2017). With this background, this study was designed to explore the bioactive molecules isolated from $S$. marginatum for its anticancer activity by in silico docking analysis targeting wnt signalling proteins, APC, $\beta$-catenin and GSK3 $\beta$. 


\section{Materials and Methods}

\section{Ligand preparation and optimization}

Twelve biomolecules isolated from $S$. marginatum were chosen for the study based on the review of literature (Solimabi et al., 1980; Venkateswarlu, and Biabani, 1995 and Rosa et al., 1999). The three dimensional structure of the molecules were retrieved from the seaweed metabolite database (www.swmd.co.in) and pubchem database. The compounds included in the analysis were Stoechospermol, 17,18-Epoxy, 5(R),16dihydroxyspat 13(14)-ene, Spatal, 5(R)hydroxy spata 13,17-diene, 5(R),18dihydroxy spata 13,16-diene, 5(R),16dihydroxy spata 13,17-diene, 5-oxo, 15,18,19trihydroxy spata 13,16- diene, 5(R),15,18(R/S), 19-tetrahydroxy spata 13,16diene, 19-acetoxy, 5(R), 15,16-trihydroxy spata 13,17-diene, 5(R), 17(S/R)-dihydroxy spata 13,18-diene, 5(R),16(S)-diacetoxyspata13,17-diene, $\quad 5(\mathrm{R}), 16(\mathrm{~S})$-dihydroxyspata13,17-diene. The chemical structure of the biomolecules is shown in figure 1.

\section{Protein preparation and optimization}

The crystal structure of target proteins APC, $\beta$-catenin and GSK3 $\beta$ were obtained from UniProtKB protein database. The ligands and crystallographic water molecules were removed from the proteins. The minimization of energy and addition of polar hydrogen ions were done by applying CHARMm force field. The 3 dimensional structure of the proteins are shown in figure 2 .

\section{In silico ADMET screening}

The compounds were screened for their ADMET (Absorption, Distribution, Metabolism, Excretion and Toxicity) properties by evaluating their drug-likeness and physicochemical properties using
Discovery Studio 4.0. The drug-likeness property of a compound was evaluated by Lipinki's rule of five. The parameters that were studied to predict the drug likeness property of the compounds were molecular weight, $\log \mathrm{P}$, hydrogen bond donors, hydrogen bond acceptors and molar refractivity.

The physicochemical parameters that were screened were solubility, blood brain barrier permeability, hepatotoxicity, plasma protein binding ability, cytochrome P450 inhibition and AMES mutagenicity.

\section{Molecular docking}

The docking analysis of ligands and target proteins were carried out using Accelrys Discovery Studio 4.0. The docking score, number of hydrogen bonds, amino acids involved in hydrogen bonding and distance of hydrogen bond were estimated.

\section{Results and Discussion}

\section{In silico ADMET screening}

The drug likeness score of the bioactive compounds from $S$. marginatum are given in table 1. All the compounds accepted Lipinski's rule of 5 and showed drug-likeness properties. Lipinski's rule of 5 is widely applied to screen compounds for druglikeness properties that could have good oral absorption and / or permeation. As per this rule, orally active drugs will have molecular mass $\leq 500, \log \mathrm{P}$ (octanol-water partition coefficient) $\leq 5$, Hydrogen bond donors $\leq 5$, Hydrogen bond acceptors $\leq 10$ and molar refractivity between $40-130$ (Kumar et al, 2016).

The predicted ADMET properties of the bioactive compounds from $S$. marginatum are given in table 2. All the compounds were 
found to be non-mutagenic as predicted by TOPKAT AMES mutagenicity. The aqueous solubility of all compounds varied from low to optimal. The aqueous solubility of the compound 7 was found to be optimal and found to be promising entity for further evaluation. The aqueous solubility of all compounds except compound 2 (spatol), 3, 10 and 12 (Stoechospermol) were found to be good. The blood brain barrier (BBB) penetration score of the compounds varied from $0-3$. Among the compounds screened compound 6, 7 and 8 showed low BBB penetrability. All other compounds showed very high to medium BBB penetrability which indicates possible CNS side effects and it would be a limiting factor.

Table.1 Lipinski's Rule of 5 parameters for the compounds isolated from S. marginatum

\begin{tabular}{|c|c|c|c|c|c|c|c|}
\hline $\begin{array}{l}\text { Comp. } \\
\text { No. }\end{array}$ & $\begin{array}{l}\text { Name of the } \\
\text { Compound }\end{array}$ & $\begin{array}{l}\text { Mol. Wt. } \\
\text { (g/mol) }\end{array}$ & $\begin{array}{l}\text { Mol. } \\
\text { Formulae }\end{array}$ & $\begin{array}{l}\text { H } \\
\text { bond } \\
\text { Donor }\end{array}$ & $\begin{array}{l}\mathrm{H} \text { bond } \\
\text { acceptor }\end{array}$ & $\begin{array}{l}\text { Molar } \\
\text { Refractivity }\end{array}$ & $\begin{array}{l}\log P< \\
5\end{array}$ \\
\hline 1 & $\begin{array}{l}\text { 17,18-Epoxy, 5(R),16- } \\
\text { dihydroxyspat 13(14)- } \\
\text { ene }\end{array}$ & 320.47 & $\mathrm{C}_{20} \mathrm{H}_{32} \mathrm{O}_{3}$ & 2 & 3 & 92.51 & 2.679 \\
\hline 2 & Spatol & 318.45 & $\mathrm{C}_{20} \mathrm{H}_{30} \mathrm{O}_{3}$ & 1 & 3 & 90.32 & 2.613 \\
\hline 3 & $\begin{array}{l}\text { 5(R)-hydroxy spata } \\
\text { 13,17-diene }\end{array}$ & 288.47 & $\mathrm{C}_{20} \mathrm{H}_{32} \mathrm{O}$ & 1 & 1 & 91.87 & 4.947 \\
\hline 4 & $\begin{array}{l}\text { 5(R),18-dihydroxy } \\
\text { spata 13,16-diene }\end{array}$ & 304.47 & $\mathrm{C}_{20} \mathrm{H}_{32} \mathrm{O}_{2}$ & 2 & 2 & 93.03 & 3.536 \\
\hline 5 & $\begin{array}{l}\text { 5(R),16-dihydroxy } \\
\text { spata 13,17-diene }\end{array}$ & 304.24 & $\mathrm{C}_{20} \mathrm{H}_{32} \mathrm{O}_{2}$ & 2 & 2 & 93.03 & 3.845 \\
\hline 6 & $\begin{array}{l}\text { 5-oxo, } 15,18,19- \\
\text { trihydroxy spata } 13,16- \\
\text { diene }\end{array}$ & 334.45 & $\mathrm{C}_{20} \mathrm{H}_{30} \mathrm{O}_{4}$ & 3 & 4 & 94.43 & 1.782 \\
\hline 7 & $\begin{array}{l}5(\mathrm{R}), 15,18(\mathrm{R} / \mathrm{S}), 19- \\
\text { tetrahydroxy spata } \\
\text { 13,16-diene }\end{array}$ & 336.46 & $\mathrm{C}_{20} \mathrm{H}_{32} \mathrm{O}_{4}$ & 4 & 4 & 95.39 & 1.681 \\
\hline 8 & $\begin{array}{l}\text { 19-acetoxy, } 5(\mathrm{R}), \\
15,16 \text {-trihydroxy spata } \\
13,17 \text {-diene }\end{array}$ & 378.50 & $\mathrm{C}_{22} \mathrm{H}_{34} \mathrm{O}_{5}$ & 3 & 5 & 105.09 & 2.302 \\
\hline 9 & $\begin{array}{l}\text { 5(R), } 17(\mathrm{~S} / \mathrm{R})- \\
\text { dihydroxy spata } 13,18- \\
\text { dien }\end{array}$ & 304.46 & $\mathrm{C}_{20} \mathrm{H}_{32} \mathrm{O}_{2}$ & 2 & 2 & 93.03 & 3.899 \\
\hline 10 & $\begin{array}{l}\text { 5(R),16(S)- } \\
\text { diacetoxyspata-13,17- } \\
\text { diene }\end{array}$ & 388.54 & $\mathrm{C}_{24} \mathrm{H}_{36} \mathrm{O}_{4}$ & 0 & 4 & 112.50 & 4.603 \\
\hline 11 & $\begin{array}{l}\text { 5(R),16(S)- } \\
\text { dihydroxyspata-13,17- } \\
\text { diene }\end{array}$ & 304.47 & $\mathrm{C}_{20} \mathrm{H}_{32} \mathrm{O}_{2}$ & 2 & 2 & 93.03 & 3.845 \\
\hline 12 & Stoechospermol & 288.47 & $\mathrm{C}_{20} \mathrm{H}_{32} \mathrm{O}$ & 1 & 1 & 91.61 & 4.901 \\
\hline
\end{tabular}


Table.2 ADMET profile of the compounds isolated from $S$. marginatum

\begin{tabular}{|c|c|c|c|c|c|c|c|}
\hline $\begin{array}{l}\text { Comp. } \\
\text { No. }\end{array}$ & $\begin{array}{l}\text { Name of the } \\
\text { Compound }\end{array}$ & $\begin{array}{l}\text { Solubility } \\
\text { Level }\end{array}$ & $\begin{array}{l}\text { BBB } \\
\text { Level }\end{array}$ & $\begin{array}{l}\text { Hepatotoxicity } \\
\text { Prediction }\end{array}$ & $\begin{array}{l}\text { CYP2D6 } \\
\text { inhibition }\end{array}$ & $\begin{array}{l}\text { PPB } \\
\text { Prediction }\end{array}$ & $\begin{array}{l}\text { AMES } \\
\text { Mutagenicity }\end{array}$ \\
\hline 1 & $\begin{array}{l}\text { 17,18-Epoxy, } 5(\mathrm{R}), 16- \\
\text { dihydroxyspat } 13(14)- \\
\text { ene }\end{array}$ & 3 & 2 & False & False & True & NM \\
\hline 2 & Spatol & 2 & 1 & False & False & True & NM \\
\hline 3 & $\begin{array}{l}\text { 5(R)-hydroxy spata } \\
\text { 13,17-diene }\end{array}$ & 2 & 0 & False & False & True & NM \\
\hline 4 & $\begin{array}{l}\text { 5(R),18-dihydroxy } \\
\text { spata 13,16-diene }\end{array}$ & 3 & 1 & False & False & True & NM \\
\hline 5 & $\begin{array}{l}\text { 5(R),16-dihydroxy } \\
\text { spata 13,17-diene }\end{array}$ & 3 & 1 & False & False & True & NM \\
\hline 6 & $\begin{array}{l}\text { 5-oxo, 15,18,19- } \\
\text { trihydroxy spata 13,16- } \\
\text { diene }\end{array}$ & 3 & 3 & False & False & True & NM \\
\hline 7 & $\begin{array}{l}5(\mathrm{R}), 15,18(\mathrm{R} / \mathrm{S}), 19- \\
\text { tetrahydroxy spata } \\
\text { 13,16-diene }\end{array}$ & 4 & 3 & False & False & False & NM \\
\hline 8 & $\begin{array}{l}\text { 19-acetoxy, } 5(\mathrm{R}), 15,16- \\
\text { trihydroxy spata } 13,17- \\
\text { diene }\end{array}$ & 3 & 3 & False & False & False & NM \\
\hline 9 & $\begin{array}{l}5(\mathrm{R}), 17(\mathrm{~S} / \mathrm{R}) \text {-dihydroxy } \\
\text { spata } 13,18 \text {-dien }\end{array}$ & 3 & 1 & False & False & True & NM \\
\hline 10 & $\begin{array}{l}5(\mathrm{R}), 16(\mathrm{~S})- \\
\text { diacetoxyspata-13,17- } \\
\text { diene }\end{array}$ & 2 & 1 & False & False & True & NM \\
\hline 11 & $\begin{array}{l}\text { 5(R),16(S)- } \\
\text { dihydroxyspata-13,17- } \\
\text { diene }\end{array}$ & 3 & 1 & False & False & True & NM \\
\hline 12 & Stoechospermol & 2 & 0 & False & False & True & NM \\
\hline
\end{tabular}

ADMET solubility Level: level 0 - extremely low, 1- very low but possible, 2 - low, 3-good, 4- optimal, 5-too soluble; ADMET BBB permeability level: Level 0 - very high penetrant, 1- high penetrant, 2-medium penetrant, 3-low penetrant 4undefined. NM- Non-mutagenic 
Table.3 Docking results of the compounds isolated from S. marginatum with $\beta$-catenin protein

\begin{tabular}{|c|c|c|c|c|c|}
\hline $\begin{array}{l}\text { Comp. } \\
\text { No. }\end{array}$ & Compound Name & $\begin{array}{l}\text { Libdock } \\
\text { score }\end{array}$ & $\begin{array}{l}\text { No. of } \\
\text { Hydrogen } \\
\text { bonds }\end{array}$ & $\begin{array}{l}\text { Amino acids } \\
\text { involved in } \\
\text { hydrogen bond }\end{array}$ & $\begin{array}{l}\text { Distance of } \\
\text { hydrogen } \\
\text { bonds }\end{array}$ \\
\hline 1 & $\begin{array}{l}\text { 17,18-Epoxy, } 5(\mathrm{R}), 16- \\
\text { dihydroxyspat } 13(14) \text {-ene }\end{array}$ & 84.033 & 2 & $\begin{array}{l}\text { ASN A:516 } \\
\text { GLU A: } 571\end{array}$ & $\begin{array}{l}2.80 \\
1.83\end{array}$ \\
\hline 2 & Spatol & 87.204 & 2 & $\begin{array}{l}\text { ASN A: } 516 \\
\text { SER A: } 473\end{array}$ & $\begin{array}{l}1.78 \\
2.09\end{array}$ \\
\hline 3 & $\begin{array}{l}\text { 5(R)-hydroxy spata } 13,17- \\
\text { diene }\end{array}$ & 82.735 & 3 & $\begin{array}{l}\text { ARG A: } 474 \\
\text { SER A: } 473 \\
\text { ASN A: } 516\end{array}$ & $\begin{array}{l}2.84 \\
1.92 \\
2.03\end{array}$ \\
\hline 4 & $\begin{array}{l}\text { 5(R),18-dihydroxy spata } \\
\text { 13,16-diene }\end{array}$ & 88.087 & 3 & $\begin{array}{l}\text { ARG A: } 474 \\
\text { GLU A: } 571 \\
\text { ASN A: } 516\end{array}$ & $\begin{array}{l}2.48 \\
1.86 \\
1.83\end{array}$ \\
\hline 5 & $\begin{array}{l}\text { 5(R),16-dihydroxy spata } \\
\text { 13,17-diene }\end{array}$ & 88.347 & 2 & $\begin{array}{l}\text { ASN A: } 516 \\
\text { SER A: } 473\end{array}$ & $\begin{array}{l}1.79 \\
1.78\end{array}$ \\
\hline 6 & $\begin{array}{l}\text { 5-oxo, } 15,18,19- \\
\text { trihydroxy spata } 13,16- \\
\text { diene }\end{array}$ & 88.856 & 5 & $\begin{array}{l}\text { ARG A: } 515 \\
\text { AGR A: } 474 \\
\text { ASN A: } 516 \\
\text { ASN A: } 516 \\
\text { SER A: } 473\end{array}$ & $\begin{array}{l}1.77 \\
2.45 \\
1.90 \\
1.92 \\
1.84 \\
\end{array}$ \\
\hline 7 & $\begin{array}{l}5(\mathrm{R}), 15,18(\mathrm{R} / \mathrm{S}), 19- \\
\text { tetrahydroxy spata } 13,16- \\
\text { diene }\end{array}$ & 98.924 & 3 & $\begin{array}{l}\text { ASN A: } 516 \\
\text { ASN A: } 516 \\
\text { GLU A: } 571\end{array}$ & $\begin{array}{l}2.45 \\
1.76 \\
1.84\end{array}$ \\
\hline 8 & $\begin{array}{l}\text { 19-acetoxy, } 5(\mathrm{R}), 15,16- \\
\text { trihydroxy spata } 13,17 \text { - } \\
\text { diene }\end{array}$ & 95.603 & 4 & $\begin{array}{l}\text { ARG A: } 474 \\
\text { ARG A: } 474 \\
\text { ARG A: } 612 \\
\text { ARG A: } 515\end{array}$ & $\begin{array}{l}2.14 \\
1.70 \\
1.74 \\
2.10 \\
\end{array}$ \\
\hline 9 & $\begin{array}{l}\text { 5(R), 17(S/R)-dihydroxy } \\
\text { spata 13,18-dien }\end{array}$ & 88.18 & 4 & $\begin{array}{l}\text { ASN A: } 516 \\
\text { SER A: } 473 \\
\text { ARG A: } 469 \\
\text { LYS A: } 508\end{array}$ & $\begin{array}{l}1.93 \\
1.88 \\
1.87 \\
2.33 \\
\end{array}$ \\
\hline 10 & $\begin{array}{l}5(\mathrm{R}), 16(\mathrm{~S})- \\
\text { diacetoxyspata-13,17- } \\
\text { diene }\end{array}$ & 96.744 & 1 & ARG A: 474 & $\begin{array}{l}1.74 \\
2.95\end{array}$ \\
\hline 11 & $\begin{array}{l}5(\mathrm{R}), 16(\mathrm{~S})- \\
\text { dihydroxyspata-13,17- } \\
\text { diene }\end{array}$ & 82.704 & 3 & $\begin{array}{l}\text { ASN A: } 474 \\
\text { ASN A: } 516 \\
\text { ARG A: } 515\end{array}$ & $\begin{array}{l}2.30 \\
1.81 \\
1.71\end{array}$ \\
\hline 12 & Stoechospermol & 79.146 & 1 & LYSA 508 & 2.22 \\
\hline
\end{tabular}


Table.4 Docking results of the compounds isolated from $S$. marginatum with APC protein

\begin{tabular}{|c|c|c|c|c|c|}
\hline $\begin{array}{l}\text { Comp. } \\
\text { No. }\end{array}$ & Name of the Compound & $\begin{array}{l}\text { Libdock } \\
\text { score }\end{array}$ & $\begin{array}{l}\text { No. of } \\
\text { Hydrogen } \\
\text { bonds }\end{array}$ & $\begin{array}{l}\text { Amino acids } \\
\text { involved in } \\
\text { hydrogen bond }\end{array}$ & $\begin{array}{l}\text { Distance of } \\
\text { hydrogen bonds }\end{array}$ \\
\hline 1 & $\begin{array}{l}\text { 17,18-Epoxy, } 5(\mathrm{R}), 16- \\
\text { dihydroxyspat } 13(14) \text {-ene }\end{array}$ & 114.579 & 1 & ARG A: 690 & 1.59 \\
\hline 2 & Spatol & 103.785 & 1 & ARG A: 690 & 2.70 \\
\hline 3 & $\begin{array}{l}\text { 5(R)-hydroxy spata } 13,17- \\
\text { diene }\end{array}$ & 105.201 & 1 & ARG A: 653 & 2.19 \\
\hline 4 & $\begin{array}{l}5(\mathrm{R}), 18 \text {-dihydroxy spata } 13,16- \\
\text { diene }\end{array}$ & 109.832 & 1 & $\begin{array}{l}\text { ARG A: } 690 \\
\text { ARG A: } 657\end{array}$ & $\begin{array}{l}1.97 \\
2.11\end{array}$ \\
\hline 5 & $\begin{array}{l}5(\mathrm{R}), 16 \text {-dihydroxy spata } 13,17 \text { - } \\
\text { diene }\end{array}$ & 110.114 & 2 & $\begin{array}{l}\text { ARG A: } 657 \\
\text { ARG A: } 657\end{array}$ & $\begin{array}{l}2.97 \\
1.91\end{array}$ \\
\hline 6 & $\begin{array}{l}\text { 5-oxo, 15,18,19-trihydroxy } \\
\text { spata } 13,16 \text { - diene }\end{array}$ & 114.594 & 2 & $\begin{array}{l}\text { ASN A: } 660 \\
\text { ARG A: } 690\end{array}$ & $\begin{array}{l}2.22 \\
1.89\end{array}$ \\
\hline 7 & $\begin{array}{l}5(\mathrm{R}), 15,18(\mathrm{R} / \mathrm{S}), 19- \\
\text { tetrahydroxy spata } 13,16 \text {-diene }\end{array}$ & 114.62 & 1 & ARG A: 657 & $\begin{array}{l}2.14 \\
1.96 \\
2.31\end{array}$ \\
\hline 8 & $\begin{array}{l}\text { 19-acetoxy, } 5(\mathrm{R}), 15,16- \\
\text { trihydroxy spata } 13,17 \text {-diene }\end{array}$ & 126.499 & 4 & $\begin{array}{l}\text { ARG A: } 653 \\
\text { ARG A: } 657 \\
\text { ALA A: } 689 \\
\text { ARG A: } 690\end{array}$ & $\begin{array}{l}1.88 \\
1.91 \\
2.04 \\
1.93\end{array}$ \\
\hline 9 & $\begin{array}{l}\text { 5(R), 17(S/R)-dihydroxy spata } \\
\text { 13,18-dien }\end{array}$ & 112.112 & 1 & ARG A: 690 & 1.78 \\
\hline 10 & $\begin{array}{l}\text { 5(R),16(S)-diacetoxyspata- } \\
\text { 13,17-diene }\end{array}$ & 109.268 & 3 & $\begin{array}{l}\text { ARG A: } 690 \\
\text { ARG A: } 653 \\
\text { ARG A: } 653\end{array}$ & $\begin{array}{l}1.71 \\
2.92 \\
1.68\end{array}$ \\
\hline 11 & $\begin{array}{l}\text { 5(R),16(S)-dihydroxyspata- } \\
\text { 13,17-diene }\end{array}$ & 99.398 & 2 & $\begin{array}{l}\text { ARG A: } 657 \\
\text { LEU A: } 684\end{array}$ & $\begin{array}{l}1.73 \\
1.72\end{array}$ \\
\hline 12 & Stoechospermol & 99.862 & 1 & ARG A: 690 & 2.36 \\
\hline
\end{tabular}


Table.5 Docking results of the compounds isolated from $S$. marginatum with glycogen synthase kinase-3 beta (GSK3 $\beta$ ) protein

\begin{tabular}{|c|c|c|c|c|c|}
\hline $\begin{array}{l}\text { Comp. } \\
\text { No. }\end{array}$ & Name of the Compound & $\begin{array}{l}\text { Libdock } \\
\text { score }\end{array}$ & $\begin{array}{l}\text { No. of } \\
\text { Hydrogen } \\
\text { bonds }\end{array}$ & $\begin{array}{l}\text { Amino acids } \\
\text { involved in } \\
\text { hydrogen bond }\end{array}$ & $\begin{array}{l}\text { Distance of } \\
\text { hydrogen } \\
\text { bonds }\end{array}$ \\
\hline 1 & $\begin{array}{l}\text { 17,18-Epoxy, 5(R),16- } \\
\text { dihydroxyspat 13(14)-ene }\end{array}$ & 99.572 & 1 & GLY A: 202 & 1.83 \\
\hline 2 & Spatol & 107.967 & 3 & $\begin{array}{l}\text { LYS A: } 85 \\
\text { ARG A: } 96 \\
\text { ASN A: } 95\end{array}$ & $\begin{array}{l}2.11 \\
2.53 \\
1.93\end{array}$ \\
\hline 3 & $\begin{array}{l}\text { 5(R)-hydroxy spata } 13,17- \\
\text { diene }\end{array}$ & 90.122 & 2 & GLU A: 97 & $2.96,2.68$ \\
\hline 4 & $\begin{array}{l}5(\mathrm{R}), 18 \text {-dihydroxy spata } 13,16- \\
\text { diene }\end{array}$ & 95.71 & 3 & $\begin{array}{l}\text { SER A: } 66 \\
\text { PHE A: } 67 \\
\text { GLU A: } 97\end{array}$ & $\begin{array}{l}2.00 \\
2.46 \\
2.75\end{array}$ \\
\hline 5 & $\begin{array}{l}5(\mathrm{R}), 16 \text {-dihydroxy spata } 13,17- \\
\text { diene }\end{array}$ & 89.27 & 2 & $\begin{array}{l}\text { ASP A: } 200 \\
\text { SER A: } 203\end{array}$ & $\begin{array}{l}1.99 \\
1.76\end{array}$ \\
\hline 6 & $\begin{array}{l}\text { 5-oxo, } 15,18,19 \text {-trihydroxy spata } \\
\text { 13,16- diene }\end{array}$ & 106.176 & 1 & $\begin{array}{l}\text { ASN A: } 95 \\
\text { GLY A: } 65\end{array}$ & $\begin{array}{l}1.94 \\
2.90\end{array}$ \\
\hline 7 & $\begin{array}{l}5(\mathrm{R}), 15,18(\mathrm{R} / \mathrm{S}), 19 \text {-tetrahydroxy } \\
\text { spata } 13,16 \text {-diene }\end{array}$ & 113.791 & 3 & $\begin{array}{l}\text { LYS A: } 85 \\
\text { GLU A: } 97 \\
\text { GLY A: } 202\end{array}$ & $\begin{array}{l}1.65 \\
1.71 \\
1.77\end{array}$ \\
\hline 8 & $\begin{array}{l}\text { 19-acetoxy, } 5(\mathrm{R}), 15,16 \text {-trihydroxy } \\
\text { spata } 13,17 \text {-diene }\end{array}$ & 123.31 & 5 & $\begin{array}{l}\text { ARG A: } 96 \\
\text { LYS A: } 94 \\
\text { GLU A: } 97 \\
\text { GLY A: } 68 \\
\text { PHE A: } 67\end{array}$ & $\begin{array}{l}1.82 \\
2.15 \\
1.83 \\
2.10 \\
2.36\end{array}$ \\
\hline 9 & $\begin{array}{l}\text { 5(R), 17(S/R)-dihydroxy spata } 13,18 \text { - } \\
\text { dien }\end{array}$ & 97.253 & 3 & $\begin{array}{l}\text { LYS A: } 85 \\
\text { ASN A: } 95 \\
\text { ASP A: } 200\end{array}$ & $\begin{array}{l}1.70 \\
2.07 \\
1.80\end{array}$ \\
\hline 10 & $\begin{array}{l}\text { 5(R),16(S)-diacetoxyspata-13,17- } \\
\text { diene }\end{array}$ & 100.864 & 1 & SER A: 203 & 1.89 \\
\hline 11 & $\begin{array}{l}\text { 5(R),16(S)-dihydroxyspata-13,17- } \\
\text { diene }\end{array}$ & 105.914 & 4 & $\begin{array}{l}\text { LYS A: } 85 \\
\text { ASN A: } 95 \\
\text { ASP A: } 200 \\
\text { ARG A: } 96\end{array}$ & $\begin{array}{l}1.60 \\
1.92 \\
2.54 \\
2.78\end{array}$ \\
\hline 12 & Stoechospermol & 84.641 & 1 & ASN A: 95 & 2.58 \\
\hline
\end{tabular}


Fig.1 Chemical structure of bioactive compounds isolated from S. marginatum
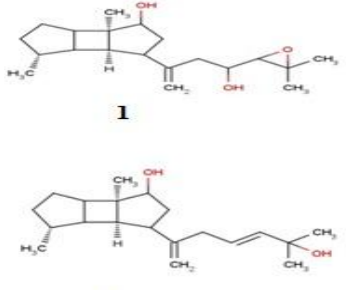

4
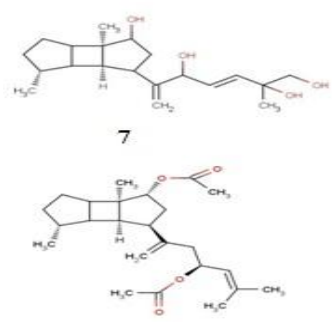

10
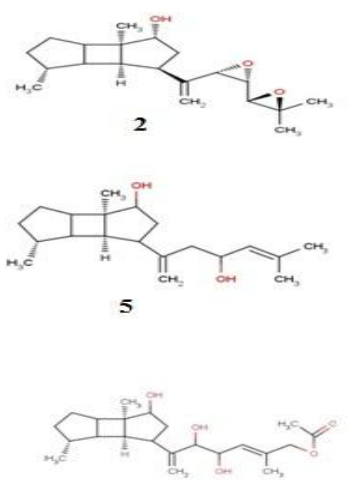

$\mathbf{8}$

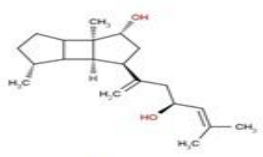

11
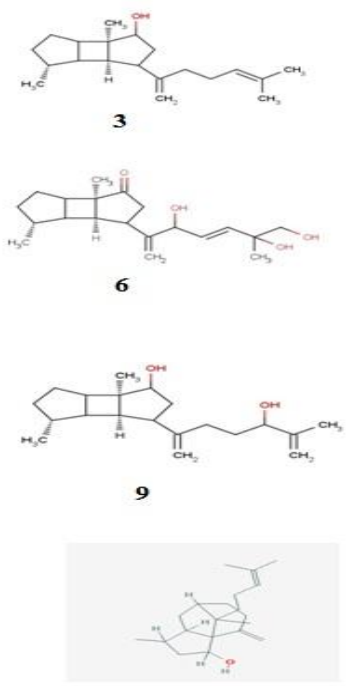

12

Fig.2 Three dimensional structure of Wnt signalling proteins a) $\beta$-catenin b) APC c) GSK3 $\beta$

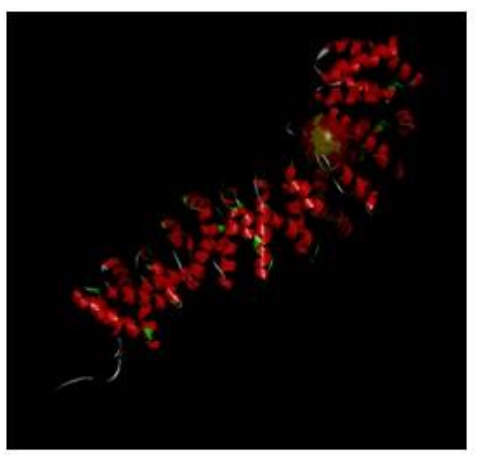

a

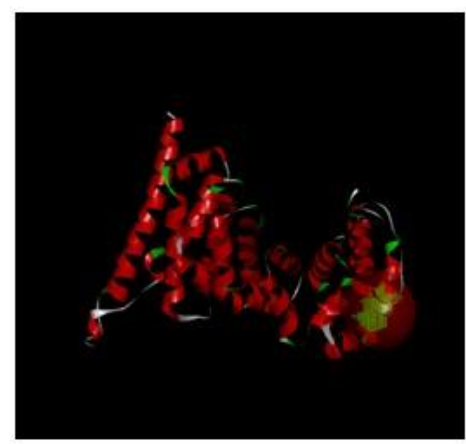

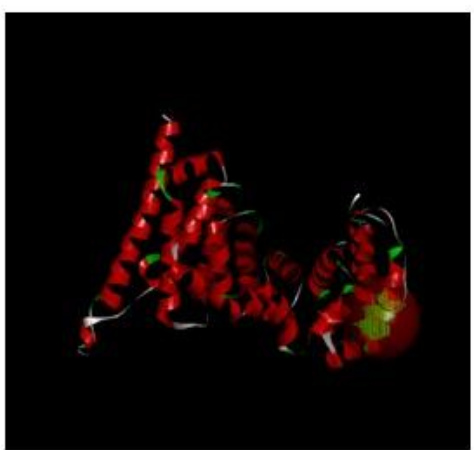

b 
Fig.3 Docking Interaction of ligands with wnt signalling proteins. $\beta$-catenin with Compound 7 (a) and Compound 8 (b). APC interaction with Compound 7 (c) and Compound 8 (d). GSK $3 \beta$ interaction with Compound 7 (e) and Compound 8 (f)

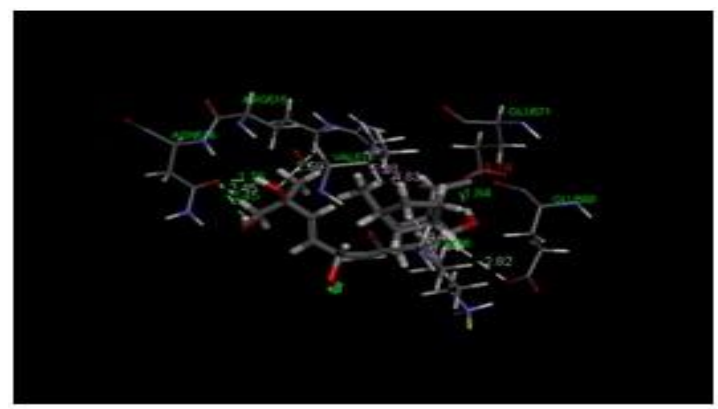

a

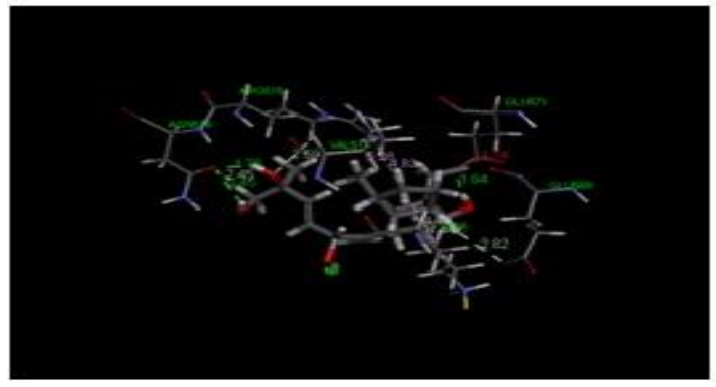

c

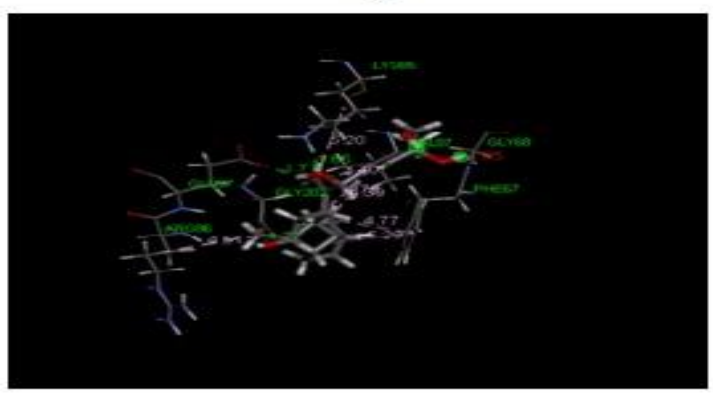

e

All the compounds screened for hepatotoxicity were found to be non-toxic. All the compounds screened were found to be non-inhibitor of CPY2D6. The cytochrome P450 2D6 is involved in the metabolism of wide range of xenobiotics and its inhibition by a drug may lead to serious drug-drug interactions (Szumilak et al., 2016). Hence, potential adverse effects resulting from drugdrug interactions of these bioactive molecules are unlikely. All the compounds tested except compounds $\mathbf{7}$ and $\mathbf{8}$ were likely to be highly bound to plasma proteins. The pharmacological activity is determined by

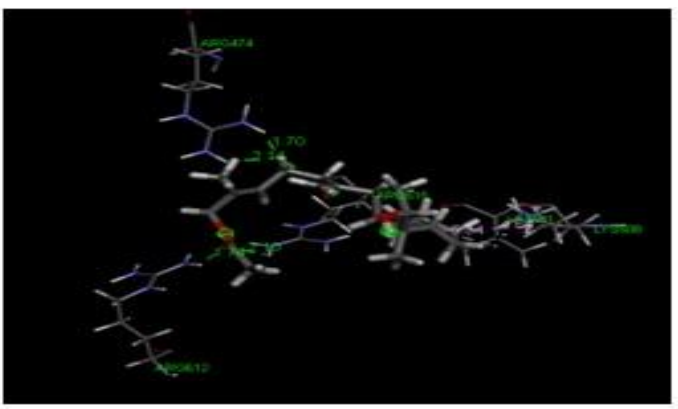

b

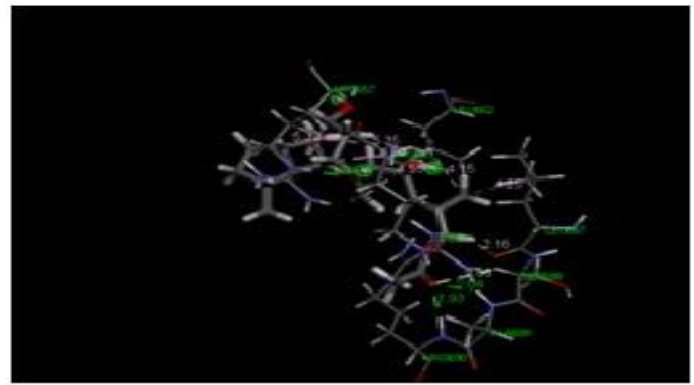

d

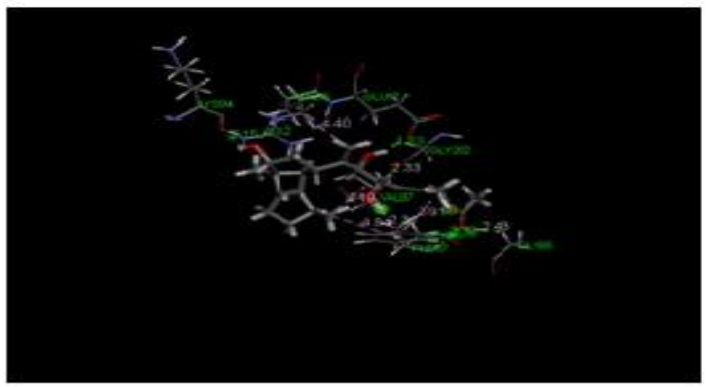

f

free plasma drug concentration and hence plasma protein binding of a compound should be taken into account during drug discovery.

\section{Docking analysis}

The docking results of the compounds with $\beta$ catenin, APC and GSK3 $\beta$ are presented in table 3, 4 and 5, respectively and in figure 3 . All the compounds docked with $\beta$-catenin protein with dock scores ranging from 79.146 to 98.924. The amino acids which are involved in interaction were AGR A: 474, ARG A: 469, ARG A: 515, ARG A: 612, 
ASN A: 516, GLU A: 571, LYS A 508 and SER A: 473. The compound 7 showed highest dock score of 98.924 with three hydrogen bond interaction with the amino acids ASN A: 516 and GLU A: 571.

All the compounds docked well with APC protein with dock score ranging from 99.398 to 126.499 . The amino acids involved in the interaction were ALA A: 689, ARG A: 653, ARG A: 657, ARG A: 690, ASN A: 660 and LEU A: 684. The compound 8 showed highest dock score of 126.499 with four hydrogen bond interaction with the amino acids ARG A: 653, ARG A: 657, ALA A: 689, ARG A: 690.

Similarly, all the compounds showed good docking results with glycogen synthase kinase-3 beta (GSK3 $\beta$ ) protein with dock score ranging from 84.641 to 123.31. The amino acids involved in the interaction were ARG A: 96, ASN A: 95, ASP A: 200, GLU A: 97, GLY A: 202, GLY A: 65, GLY A: 68, LYS A: 85, LYS A: 94, PHE A: 67, SER A: 203, SER A: 66. Among the compounds screened, compound 8 showed highest dock score of 123.31 with 5 hydrogen bond interaction with the amino acid residues ARG A: 96, LYS A: 94, GLU A: 97, GLY A: 68 and PHE A: 67

In silico docking analysis of the bioactive compounds from $S$. marginatum revealed that the screened compounds were found to potentially inhibit Wnt related downstream targeted proteins of CRC, APC, $\beta$-catenin and GSK3 $\beta$.

The compound $7(5(\mathrm{R}), 15,18(\mathrm{R} / \mathrm{S}), 19$ tetrahydroxy spata 13,16-diene) and compound 8 (19-acetoxy, 5(R), 15, 16trihydroxy spata 13,17 -diene) showed very good interaction with $\beta$-catenin, APC and GSK3 $\beta$ proteins. In addition, compound 7 and 8 met required ADMET criteria with good aqueous solubility, low BBB penetrability, low plasma protein binding, non-hepatotoxic, non-mutagenic and lack of CYP2D6 inhibition. Among the bioactive compounds screened, compound 7 and 8 were found to be promising and it would be a valuable lead candidate for the further development of drugs against colorectal cancer.

\section{References}

Anbu, A., Arun, E., Selvakumari, E. and Gopal, V. 2017. Phytochemical and pharmacological profile of brown marine algae Stoecheospermum marginatum. Journal of Academia and Industrial Research, 5(8): 120-122

Arnold, M., Sierra, M.S., Laversanne, M., Soerjomataram, I., Jemal, A. and Bray, F.V 2016. Global patterns and trends in colorectal cancer incidence and mortality. Gut, Pp. 1-9

Becer, E., Hanoglu, D. Y., Kabaday, H., Hanoglu, A., Vatansever, S. Yavuz, D. O., Mericli, F. and Mericli, A.H. 2019. The effect of Colchicum pusillum in colon cancer cells via Wnt/ $\beta$-catenin pathway. Gene, 686: 213 - 219 .

Blaj, C., Bringmann, A., Schmidt, E.M., Urbischek, M., Lamprecht, S., Frohlich, T., Arnold, G.J., Krebs, S., Blum, H., Hermeking, H., Jung, A. Kirchner, T. and Horst, D. 2017. ADNP is therapeutically inducible repressor of WNT signaling in colorectal cancer. Clin Cancer Research, 23(11): 2769-2780

Bray, F., Ferlay, J., Soerjomataram, I., Siegel, R.L., Torre, L.A. and Jemal, A. 2018. Global Cancer Statistics 2018: GLOBOCAN Estimates of Incidence and Mortality Worldwide for 36 Cancers in 185 Countries. Cancer J Clin., 68: 394-424

Cheng, X., Xu, X., Chen, D., Zhao, F. and 
Wang, W. 2019. Therapeutic potential of targeting of Wnt/ $\beta$-catenin signaling pathway in colorectal cancer. Biomedicine and Pharmacotherapy, 110: 473-481

Haniya, A.M.K., Sweety, F.Y., Kothai, S. and Mahalakshmi, K. 2015. Antibacterial activity of Chaetomorpha litorea (Harvey) against isolated fish bacteria. Indian Journal of Geo-marine Science, 44(3): $1-5$

Kumar, N., Mishra, S.S., Sharma, C.S. and Singh, H.P.2016. In silico ADME, Bioactivity and Toxicity analysis of some selected antimalarial agents. International journal of Applied Pharmaceutical and Biological Research, 1(5): 1-8

Navaneethakrishnan, P., Prashantha, C.N., Boopathi, S., Sabitha, R. and Mathan G., 2013. In Silico design of Butea monosperma floral derived compounds and its inhibitory effect on $\beta$-catenin, GSK-3 $\beta$ and APC complex proteins in colorectal cancer. International Journal of Drug Discovery, 5(1): 191-197

Novellasdemunt, L., Antas, P. and Li, V.S.W. 2015. Targeting wnt signaling in colorectal cancer. A Review in the Theme: Cell Signaling: Proteins, Pathways and Mechanisms. Am J Physiol Cell Physiol, 309: C511-C521

Rosa, S., Iodice, C., Khalaghdoust, M. Oryan, S. and Rustaiyan, A. 1999. Spatane diterpenoids from the brown alga Stoechospermum marginatum (Dictyotaceae). Phytochemistry, 51(8): 1009-1012

Solimabi, Fernandes, L., Kamat, S.Y. and Paknikar, S.K. 1980. New diterpenoids of the brown seaweed Stoechospermum marginatum: structure of stoechospermol. Tetrahedron Letters, 21(23): 22492252.

Szumalik, M., Lewgowd, W. and Stanczak, A. 2016. In silico ADME studies of polyamine conjugates as potential anticancer drugs. Acta Poloniae Pharmaceutica, 73(5): 191-1200

Venkateswarlu, Y., and Biabani, F. 1995. A spatane diterpene from the brown alga Stoechospermum marginatum. Phytochemistry, 40(1): 331-333

Zhan, T., Rindtorff, N. and Boutros, M. 2017. Wnt signaling in cancer. Oncogene, 36: $1461-1473$

\section{How to cite this article:}

Kalaiselvi, L., P. Sriram, S.P. Preetha, M. Parthiban and Kannan, T.A. 2019. In silico Docking Analysis of Bioactive Compounds from Stoechoespermum marginatum against Colorectal Cancer Int.J.Curr.Microbiol.App.Sci. 8(05): 1347-1358. doi: https://doi.org/10.20546/ijcmas.2019.805.154 